\title{
Outcomes after total robotic esophagectomy for esophageal cancer: a propensity-matched comparison with hybrid robotic esophagectomy
}

\author{
Kwon Joong Na, Samina Park, In Kyu Park, Young Tae Kim, Chang Hyun Kang \\ Department of Thoracic and Cardiovascular Surgery, Seoul National University Hospital, Seoul, Republic of Korea \\ Contributions: (I) Conception and design: KJ Na, CH Kang; (II) Administrative support: CH Kang; (III) Provision of study materials of patients: \\ CH Kang; (IV) Collection and assembly of data: KJ Na, CH Kang; (V) Data analysis and interpretation: KJ Na, CH Kang; (VI) Manuscript writing: \\ All authors; (VII) Final approval of manuscript: All authors. \\ Correspondence to: Chang Hyun Kang, MD, PhD. Department of Thoracic and Cardiovascular Surgery, Seoul National University Hospital, 101 \\ Daehak-ro, Jongno-gu, Seoul, Republic of Korea. Email: chkang@snu.ac.kr.
}

Background: Robot-assisted minimally invasive esophagectomy (RAMIE) reduces postoperative respiratory complications and enables meticulous mediastinal lymphadenectomy. However, whether adding a robotic abdominal procedure to a robotic thoracic procedure can result in better outcomes is unclear. We examined outcomes after total-RAMIE (T-RAMIE) and compared them with the outcomes after hybridRAMIE (H-RAMIE).

Methods: Total of 227 patients who underwent robotic esophagectomy for esophageal cancer were included. T-RAMIE was defined as esophagectomy performed robotically in both the thoracic and abdominal cavities. Laparotomy was used instead of the robotic procedure in H-RAMIE. T-RAMIE was performed in 144 patients (63.4\%), and propensity score matching produced 49 matched pairs from each group. Early and long-term clinical outcomes between the two groups were compared.

Results: T-RAMIE was mostly performed for upper or mid-thoracic squamous cell carcinoma ( $\mathrm{n}=119$, $82.6 \%)$ and cervical anastomosis, and three-field lymphadenectomy was performed in $113(78.5 \%)$ and 54 (37.5\%) patients, respectively. One laparotomy conversion was necessary because of severe obesity. The propensity-matched analysis demonstrated that T-RAMIE showed a comparable 90-day mortality rate with H-RAMIE ( $0 \%$ vs. $6.1 \%, \mathrm{P}=0.083)$. The incidence rates of total $(63.3 \%$ vs. $63.3 \% ; \mathrm{P}=1.000)$, abdominal $(8.2 \%$ vs. $14.3 \%$; $\mathrm{P}=0.366)$, and respiratory complications $(10.2 \%$ vs. $10.2 \% ; \mathrm{P}=1.000)$ were not different between two groups. The number of harvested abdominal lymph nodes was similar (12.4 49.0 vs. $12.3 \pm 8.9 ; \mathrm{P}=0.992)$. Median follow-up duration for T-RAMIE and H-RAMIE was 16.3 and 23.5 months, respectively. Two-year overall survival rate $(86.2 \%$ in T-RAMIE $v$ s. $77.6 \%$ in H-RAMIE; $\mathrm{P}=0.150)$ and recurrence-free survival (76.6\% in T-RAMIE vs. 62.2\% in H-RAMIE; $\mathrm{P}=0.280$ ) were comparable between the two groups.

Conclusions: In this matched analysis, T-RAMIE and H-RAMIE showed comparable early outcomes and long-term survival. The low tendencies of early mortality and conversion rate of T-RAMIE suggest that it might be a safe alternative to open stomach mobilization and abdominal lymphadenectomy.

Keywords: Robotic surgery; esophageal surgery; minimally invasive surgery; clinical outcomes

Submitted Jul 01, 2019. Accepted for publication Nov 12, 2019.

doi: $10.21037 /$ jtd.2019.11.58

View this article at: http://dx.doi.org/10.21037/jtd.2019.11.58

(c) Journal of Thoracic Disease. All rights reserved. 


\section{Introduction}

Surgical resection with radical lymphadenectomy is regarded as a standard treatment for localized esophageal cancer. The major concern of surgical resection for esophageal cancer is high postoperative mortality and morbidity rates, as the surgery involves both thoracic and abdominal cavities and requires complex procedures $(1,2)$. To improve the operative outcomes, minimally invasive esophagectomy (MIE) has been increasingly used for the treatment of esophageal cancer over the past decade. Several previous studies have demonstrated that MIE can reduce intraoperative blood loss, postoperative pulmonary complications, and the length of hospital stay with comparable short- and long-term oncologic outcomes to those of open esophagectomy (OE) (3-6).

Since robotic surgical systems were introduced in early 2000 to overcome the technical pitfalls of conventional minimally invasive surgery, robot-assisted minimally invasive esophagectomy (RAMIE) has been considered another surgical option for MIE. Robotic surgery has several technical advantages over conventional minimally invasive surgery; it can facilitate complex, minimally invasive procedures with a $10 \times$ magnified, threedimensional field of view, and it also provides the articulated instruments that can facilitate dissection with seven degrees of freedom. Previous studies have reported that RAMIE is a safe and feasible surgical technique with comparable short-term outcomes compared to conventional MIE and improved short-term outcomes compared to OE (7-11). Moreover, van der Sluis et al. reported that RAMIE also provided favorable oncologic outcomes at long-term follow up (12). Specifically, most reports have focused on robot-assisted transthoracic procedures and demonstrated additional advantages in meticulous mediastinal lymph node (LN) dissection, including dissection of the recurrent laryngeal nerve $\operatorname{LN}(7-9,13,14)$. While the clinical benefit of minimally invasive thoracic procedures has been well demonstrated in the previous studies, the effect of minimally invasive abdominal procedures in esophageal surgery has been rarely reported. A recent randomized study reported better perioperative outcomes as well as the comparable long-term oncologic outcomes of hybrid MIE with laparoscopic surgery compared to OE (15). However, whether adding a robotic abdominal procedure to a robotic thoracic procedure can result in better short- or long-term outcomes is unclear.

The aim of this study was to compare short- and long- term clinical outcomes between total RAMIE (T-RAMIE) and hybrid RAMIE (H-RAMIE) to verify any clinical benefits of the robotic abdominal procedure in the surgical treatment of esophageal cancer. Propensity-score matched analysis was performed to minimize selection bias and to account for heterogeneity between groups.

\section{Methods}

The study protocol was reviewed by the Institutional Review Board and approved as a minimal risk retrospective study (Approval No. H-1905-137-1035) that did not require individual consent according to the institutional guidelines for consent waivers.

\section{Patients}

A retrospective review of the prospectively collected database was performed for 227 patients who underwent RE from 2008 to 2018 in our institution. Among them, we excluded patients who underwent trans-hiatal esophagectomy $(\mathrm{n}=2)$, who underwent laparoscopic surgery $(\mathrm{n}=1)$, and who received the colon as a substitute graft $(n=10)$; conclusively, a total of 214 patients were included in the analysis. T-RAMIE was defined as robot-assisted surgery for both the thoracic and abdominal cavities. H-RAMIE was defined as robot-assisted esophagectomy with abdominal procedures performed by laparotomy. T-RAMIE was performed in 144 patients (63.4\%), and one laparotomy conversion was necessary due to severe obesity. H-RAMIE was performed in 70 patients (36.6\%), and three thoracotomy conversions were necessary due to severe pleural adhesion.

\section{Operative technique}

We used a four-arm technique for both the thoracic and abdominal procedures of RAMIE. For the thoracic procedure, the patient was placed in the left semi-lateral decubitus position. We made a 4 -cm working window on the $4^{\text {th }}$ intercostal space at the mid-axillary line for both the number 1 robot arm and the assistant. The number 3 robot port was made at the $2^{\text {nd }}$ intercostal space at the mid-axillary line, the camera port was made at $7^{\text {th }}$ intercostal space at the posterior axillary line, and the number 2 robot port was made at the $10^{\text {th }}$ intercostal space between the camera port and the vertical line of the scapula tip. The robotic cart was driven over the patient's right shoulder. A monopolar scissor 
or harmonic scalpel was placed into arm number 1, the bipolar retractor into arm number 2, and cadiere forceps into arm number 3. LN dissection was performed of the whole mediastinal nodal stations. Right and left recurrent laryngeal nerve dissections were performed precisely by completely exposing the nerves and removing whole lymphatic tissues up to the thoracic inlet and contralateral hilum.

For abdominal procedures of RAMIE, the patient was placed in the supine position. A midline 4-cm sized small laparotomy was placed around the umbilicus for the working window, and the camera was placed into this window. The robotic $8 \mathrm{~mm}$ ports were then placed in the right mid-clavicular line, the left mid-clavicular line and at the left costal margins considering that the minimal distance between the ports should be more than $8 \mathrm{~cm}$. A $12 \mathrm{~mm}$ port was placed between the right mid-clavicular line and the working window for the assistant. Sequential applications of the gastrointestinal stapler were provided by the assistant, and the gastric conduit formation was fully completed in an intracorporeal manner. The pylorus-draining procedure was performed in most patients, and a feeding jejunostomy was performed in all patients. For open abdominal procedures, all procedures were performed via median laparotomy. The same stapling devices and suture materials were used for gastric conduit formation, and a feeding jejunostomy was performed in all patients.

\section{Preoperative evaluation and postoperative follow-up}

All patients underwent intensive preoperative evaluation, including upper gastrointestinal endoscopy, endoscopic ultrasound, chest computed tomography (CT), abdominal CT, positron emission tomography/CT, pulmonary function test, and blood testing. Bronchoscopy and cervical ultrasound were performed if indicated. For the assessment of recurrent laryngeal nerve injury, vocal cord function was assessed by nasal laryngoscopy on the 3 rd postoperative day in all patients. Postoperative surveillance of recurrence was conducted intensively. A positron emission tomography/CT scan was performed at 1,2 , and 5 years postoperatively, and a chest CT scan was performed at 6 months, 18 months, 3 years, and 4 years postoperatively. Endoscopic examinations were performed annually.

\section{Definition of assessment parameters}

Dissected LN groups were labeled according to the AJCC
LN classification (16), and lymph nodes from the thoracic cavity were classified using three mediastinal groups. The upper mediastinal LNs were defined as $2 \mathrm{R}$ (right upper paratracheal nodes), 4R (right lower paratracheal nodes), $2 \mathrm{~L}$ (left upper paratracheal nodes), 4L (left lower paratracheal nodes), 3P (posterior mediastinal nodes), 5 (aortopulmonary nodes), and $8 \mathrm{U}$ (upper paraesophageal nodes), as well as the LNs along the right recurrent laryngeal nerve. The middle mediastinal LNs were defined as 7 (subcarinal nodes), $8 \mathrm{M}$ (middle paraesophageal nodes), 10L (left tracheobronchial nodes), and 10R (right tracheobronchial nodes), and the lower mediastinal LNs were defined as $8 \mathrm{~L}$ (lower paraesophageal nodes), 9 (pulmonary ligament nodes), and 15 (diaphragmatic nodes).

The dysphagia grade was scored from 0 to 4 using the scoring system proposed by Mellow and Pinkas (17). Performance status was graded from 0 to 5 according to the European clinical oncology group performance status scoring system (18). Postoperative morbidity was prospectively recorded during bi-monthly morbidity conferences, and severities of complications were evaluated and graded using the Clavien-Dindo classification (19) and the Esophagectomy Complications Consensus Group (ECCG) classifications (20).

\section{Statistical analysis}

All statistical analyses were performed using the $\mathrm{R}$ software package, version 3.4.3 (http://www.R-project.org). Student's $t$-test and the Wilcoxon rank-sum test were used to compare continuous variables, and the chi-square test and Fischer's exact test were used to compare nominal variables for unmatched data. Propensity scores were calculated by logistic regression modelling, including the following variables that might be considered as determinant factors in selecting surgical approaches and affecting patients' clinical outcomes: age, sex, body mass index, history of abdominal surgery, clinical $\mathrm{T}$ stage, clinical $\mathrm{N}$ stage, the presence of liver cirrhosis, history of neoadjuvant therapy for esophageal cancer, location of tumor, and cervical LN dissection. We matched propensity scores one to one with the use of the nearest neighbor methods without replacement, using a 0.15 caliper width. After the matching procedure, 49 patients were selected for each group for the analysis. The McNemar test and paired t-test were used for comparison. Overall and recurrence-free survival rates were estimated with the Kaplan-Meier method, and the differences in survival rates between the two groups were compared by 
the log-rank test. All statistical analyses were performed using the two-sided method. Statistical significance was considered with $\mathrm{P}$ values of less than 0.05 .

\section{Results}

\section{Baseline characteristics and operative details of the study population}

The demographic and clinical characteristics of the study population are summarized in Table 1. The T-RAMIE group included more patients with a smoking history and fewer patients with a history of liver cirrhosis. The T-RAMIE group included patients with better performance status with marginal significance. Patients with more advanced stages were included in the H-RAMIE group (clinical $\mathrm{T}$ stage; $\mathrm{P}=0.018$, clinical $\mathrm{N}$ positive; $\mathrm{P}=0.006$ ). Moreover, the H-RAMIE group included more patients with a previous history of abdominal operation $(\mathrm{P}<0.001)$. The esophagogastric anastomosis was performed at the cervical level in most of the patients in both groups $(73.5 \%$ in T-RAMIE $v$ s. $79.5 \%$ in H-RAMIE group; $\mathrm{P}=0.467$ ). Cervical LN dissection was performed in 54 patients (37.5\%) and 19 patients $(27.1 \%)$ in the T-RAMIE and H-RAMIE groups, respectively. Most of the patients underwent reconstruction of the esophagus through the posterior mediastinal route (100.0\% in T-RAMIE $v s .97 .1 \%$ in H-RAMIE; $\mathrm{P}=0.106$ ) (Table 2). In the matched groups, there was no difference in the baseline characteristics or operative details between the two groups (Tables 1,2).

\section{Pathological data}

In terms of the pathological stage, the patients with more advanced stage underwent H-RAMIE, and this trend was maintained after the matching process (Table 3). The total number of harvested LNs was not different between the matched groups. Furthermore, there was no difference in the number of harvested lymph nodes when we analyzed the data separately according to the lymph node stations (Table 3).

\section{Early and mid-term clinical outcomes}

There were no cases of 30- or 90-day mortality in the T-RAMIE group. In the H-RAMIE group, the 30 - and 90 -day mortality rates were $2.0 \%$ and $6.1 \%$, respectively, and there were no significant differences in mortality rates between the matched groups. The overall complication rate was $63.3 \%$, and the rate of major complications (ClavienDindo classification grade $\geq 3$ ) was $12.2 \%$ in both matched groups. Specifically, the rates of respiratory complications and leakage were not different between the matched groups $(\mathrm{P}=1.000$ and 0.480 for respiratory complications and leakage, respectively). The overall rate of abdomen-related complications was higher in the H-RAMIE group, but there was no statistical significance $(\mathrm{P}=0.366)$. The median length of hospital stay was 14 days in both groups (Table 4).

The median follow-up period was 16.3 (0.3-95.2) months and $23.5(0.3-86.8)$ months in the T-RAMIE and H-RAMIE groups, respectively. The 2 -year overall survival rate was $86.2 \%$ in the T-RAMIE group and $77.6 \%$ in the H-RAMIE group $(\mathrm{P}=0.150$; Figure $1 A)$, and the two-year recurrencefree survival was $76.6 \%$ in the T-RAMIE group and $62.2 \%$ in the H-RAMIE group $(\mathrm{P}=0.280$; Figure $1 B)$. Both groups showed comparable overall and recurrence-free survival without significant differences.

We examined the proportion of patients with locoregional recurrence in the abdominal area and compared it between the matched groups. There were four patients in the T-RAMIE group (8.2\%) and three patients in the H-RAMIE group (6.1\%) with locoregional recurrence in the abdominal area, and no statistical significance was identified between the two groups $(\mathrm{P}=0.706)$.

\section{Discussion}

In the present study, we compared early and longterm clinical outcomes of T-RAMIE and H-RAMIE for esophageal cancer by propensity score matching using preoperative and perioperative variables. Both groups showed comparable early clinical outcomes in terms of 30-, 90-day mortality, overall and major complication rates, and length of hospital stay. The number of harvested LNs was also similar between the two groups. Long-term overall and recurrence-free survival was also similar between the two groups.

MIE is currently a well-adopted method for surgical resection of esophageal cancer, as it can reduce postoperative morbidities and improve quality of life. Due to these potential advantages, MIE has been increasingly implemented in recent decades, and the recent advancement of robotic technology has made RE another surgical option for MIE. Some previous studies have demonstrated early and long-term RE results. The reported incidences of pulmonary complications and early mortality after RE are $10-20 \%$ and $2-10 \%$, 
Table 1 Baseline and clinical characteristics

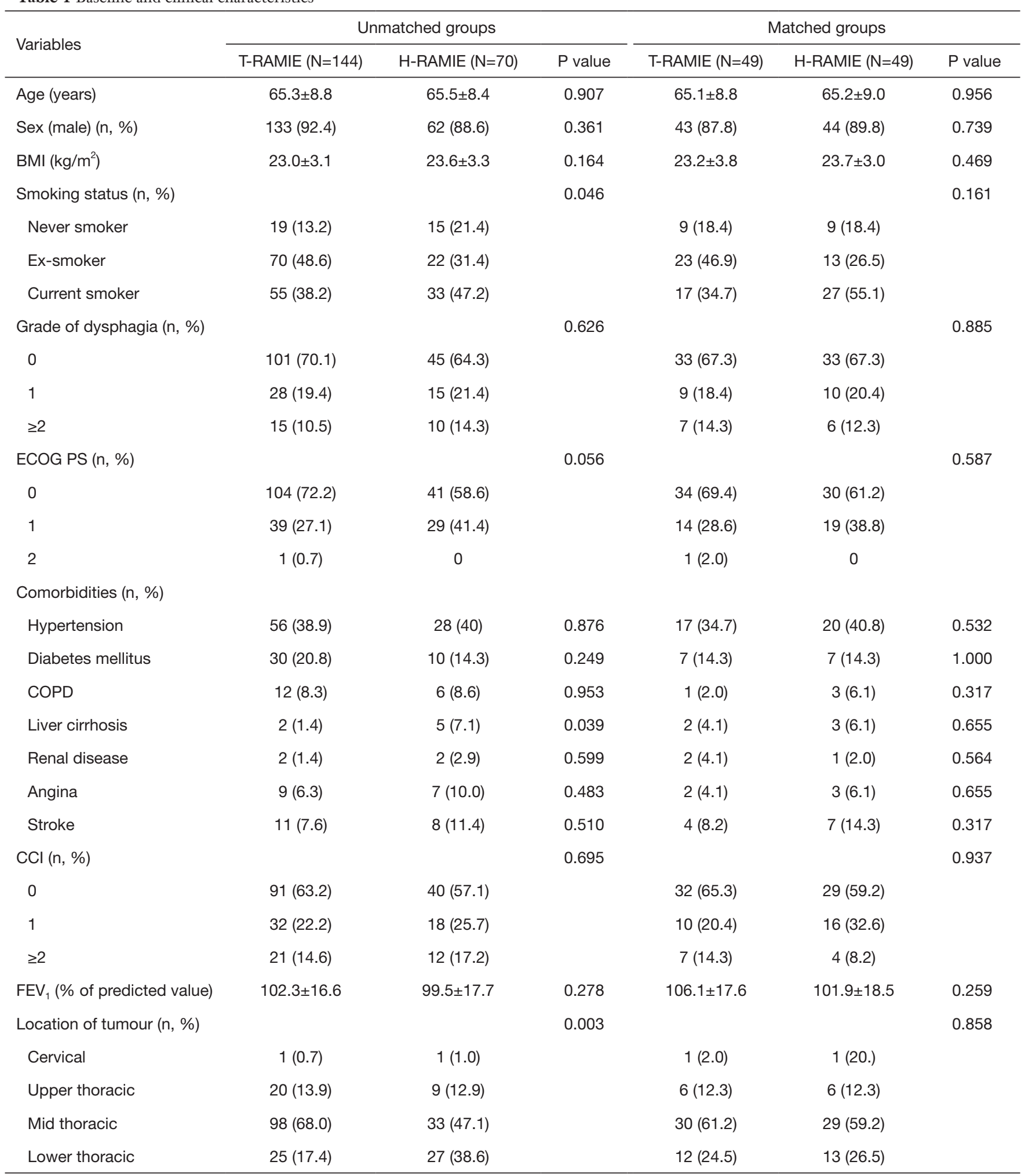

Table 1 (continued) 
Table 1 (continued)

\begin{tabular}{|c|c|c|c|c|c|c|}
\hline Variables & \multicolumn{3}{|c|}{ Unmatched groups } & \multicolumn{3}{|c|}{ Matched groups } \\
\hline Clinical T stage (n, \%) & & & 0.018 & & & 0.743 \\
\hline 1 & $69(47.9)$ & $24(34.3)$ & & $24(49.0)$ & $21(42.9)$ & \\
\hline 2 & $50(34.7)$ & $22(31.4)$ & & $11(22.4)$ & $15(30.6)$ & \\
\hline Clinical $N$ stage $(n, \%)$ & & & 0.006 & & & 0.275 \\
\hline 0 & $98(68.1)$ & $34(48.6)$ & & $31(63.3)$ & $26(53.1)$ & \\
\hline $\mathrm{N}+$ & $46(31.9)$ & $36(51.4)$ & & $18(36.7)$ & $23(46.9)$ & \\
\hline Neoadjuvant treatment (n, \%) & $35(24.3)$ & $25(35.7)$ & 0.081 & $14(28.6)$ & $16(32.7)$ & 0.637 \\
\hline RTx only & $1(0.7)$ & 0 & & 0 & 0 & \\
\hline $\begin{array}{l}\text { Previous thoracic operation } \\
(\mathrm{n}, \%)\end{array}$ & $2(1.4)$ & 0 & 1.000 & 0 & 0 & NA \\
\hline $\begin{array}{l}\text { Previous abdominal } \\
\text { operation }(n, \%)\end{array}$ & $5(3.5)$ & $12(17.1)$ & $<0.001$ & $5(10.2)$ & $3(6.1)$ & 0.414 \\
\hline
\end{tabular}

T-RAMIE, totally robot-assisted minimally invasive esophagectomy; H-RAMIE, hybrid robot-assisted minimally invasive esophagectomy; BMI, body mass index; ECOG PS, Eastern Cooperative Oncology Group performance status; COPD, chronic obstructive pulmonary disease; CCI, Charlson-Deyo comorbidity index; FEV1, forced expiratory volume in the first second; CCRT, concurrent chemoradiation therapy; CTx, chemotherapy; RTx, radiotherapy.

respectively $(7,9,11)$. van der Sluis et al. recently reported that a randomized controlled trial comparing T-RAMIE and OE, and T-RAMIE resulted in a lower incidence of postoperative complications with a better quality of life and acceptable long-term oncologic outcomes (10). Our study demonstrated that both T-RAMIE and H-RAMIE showed rates of pulmonary complications as well as early mortality similar to those in previous observations, and long-term overall and recurrence-free survival was in concordance with the current highest standards.

Most studies that evaluated the clinical outcomes of MIE examined patients who underwent minimally invasive transthoracic procedures. Specifically, many studies reported the advantages of meticulous dissection of the mediastinal LNs, including the recurrent laryngeal LNs, as robotic surgical systems provide high-definition three-dimensional visualization, motion scaling, tremor filtration, and more degrees of freedom through articulating surgical instruments $(7,9,10,21)$. Our group previously reported a significantly increased number of harvested LNs by RE compared to conventional MIE, especially in upper mediastinal lymph nodes with similar early clinical outcomes (9). Notably, the rate of recurrent laryngeal nerve injury was lower in RE. Chao et al. (21) also revealed that $\mathrm{RE}$ resulted in a higher $\mathrm{LN}$ yield along the recurrent laryngeal nerve without increasing morbidity. All patients included in our study had undergone a thoracic procedure by robotic surgery, and the overall early clinical outcome and long-term oncologic outcome were similar to those from previous studies. The respiratory complication rate was $9.3 \%$, and the 90 -day mortality rate was $1.9 \%$. A total of 26 mediastinal LNs were harvested in our study.

While the clinical advantages of MIE for the thoracic phase of esophagectomy have been well demonstrated in previous literature, there are very few reports that have evaluated the effects on clinical outcomes after minimally invasive abdominal surgery for esophageal cancer. The benefits of minimally invasive abdominal surgery have been 
Table 2 Operative details

\begin{tabular}{|c|c|c|c|c|c|c|}
\hline Variables & \multicolumn{3}{|c|}{ Unmatched groups } & \multicolumn{3}{|c|}{ Matched groups } \\
\hline Thorax operation type & & & 0.553 & & & NA \\
\hline Robot & 141 (97.9) & $70(100.0)$ & & 49 (100.0) & $49(100.0)$ & \\
\hline Open & $3(2.1)$ & 0 & & 0 & 0 & \\
\hline Cervical & $113(78.5)$ & $55(78.6)$ & & $36(73.5)$ & $39(79.6)$ & \\
\hline Thoracic & $31(21.5)$ & $15(21.4)$ & & $13(26.5)$ & $10(20.4)$ & \\
\hline Anastomosis method & & & 0.137 & & & 0.215 \\
\hline EEA & $4(2.8)$ & $2(2.9)$ & & $2(4.1)$ & $1(2.0)$ & \\
\hline Tri-staple method & $78(54.2)$ & $30(42.9)$ & & $25(51.0)$ & $21(42.9)$ & \\
\hline Route of reconstruction & & & 0.106 & & & NA \\
\hline Posterior mediastinum & $144(100.0)$ & $68(97.1)$ & & 49 (100.0) & $49(100.0)$ & \\
\hline Substernal & 0 & $2(2.9)$ & & 0 & 0 & \\
\hline Cervical LN dissection & $54(37.5)$ & $19(27.1)$ & 0.134 & $15(30.6)$ & $13(26.5)$ & 0.617 \\
\hline
\end{tabular}

T-RAMIE, totally robot-assisted minimally invasive esophagectomy; H-RAMIE, hybrid robot-assisted minimally invasive esophagectomy; LN, lymph node; NA, not applicable.

reported in gastric cancer patients. One study that compared laparoscopy and open gastrectomy showed that the major complication rate was significantly lower in the laparoscopy group (22). Furthermore, one multi-center, randomized controlled study revealed that laparoscopic surgery resulted in a lower overall complication rate with comparable intra-abdominal complications and early mortality rates compared to the open procedure (23). The robotic abdominal surgery for gastric cancer also demonstrated similar outcomes compared to laparoscopic surgery (24). For patients with esophageal cancer, a randomized controlled trial that compared OE and hybrid MIE with minimally invasive abdominal surgery was recently published (15). They showed that compared to OE, hybrid MIE for the abdominal phase of the procedure resulted in a lower incidence of major perioperative complications without compromising long-term oncologic survival. Moreover, only $3 \%$ of patients underwent open conversion. Our study revealed that T-RAMIE and H-RAMIE resulted in similar complications, mortality rates, and long-term oncologic outcomes. In particular, there was a trend of a lower abdomen-related complication rate in T-RAMIE than in H-RAMIE, and the number of harvested abdominal LNs was comparable to that in the open procedure. Moreover, there was only one laparotomy conversion case in our study. The Kaplan-Meier survival curve showed that the T-RAMIE group had a better outcome; however, it is probably because patients with more advanced esophageal cancer were included in the H-RAMIE group, even after propensity score matching. Consistent with previous observations, robotic abdominal procedures in esophageal cancer patients were relatively safe and oncologically sound alternatives to open procedures.

Although Mariette et al. (15) demonstrated the clinical benefit of hybrid MIE with the laparoscopic procedure, T-RAMIE did not show favorable early clinical outcomes compared to H-RAMIE in our study. As our study designed to compare the clinical outcomes between T-RAMIE and H-RAMIE to examine the benefit of adding a minimally invasive abdominal procedures to thoracic procedures, all patients had been benefited from a robotic thoracic procedure which is well known to improve peri-operative 
Table 3 Pathological details

\begin{tabular}{|c|c|c|c|c|c|c|}
\hline Variables & \multicolumn{3}{|c|}{ Unmatched groups } & \multicolumn{3}{|c|}{ Matched groups } \\
\hline Histology & & & 0.585 & & & NA \\
\hline Squamous cell carcinoma & $139(96.5)$ & $67(95.7)$ & & $49(100.0)$ & $49(100.0)$ & \\
\hline Adenocarcinoma & $1(0.7)$ & $2(2.9)$ & & 0 & 0 & \\
\hline Adenoidcystic carcinoma & $1(0.7)$ & 0 & & 0 & 0 & \\
\hline Melanoma & $1(0.7)$ & $1(1.4)$ & & 0 & 0 & \\
\hline GIST & $1(0.7)$ & 0 & & 0 & 0 & \\
\hline IA & $8(5.6)$ & $4(5.7)$ & & $4(8.2)$ & $3(6.1)$ & \\
\hline IB & $54(37.5)$ & $12(17.1)$ & & $15(30.6)$ & $10(20.4)$ & \\
\hline$\| \mathrm{A}$ & $9(6.3)$ & $5(7.1)$ & & $4(8.2)$ & $3(6.1)$ & \\
\hline IIB & $10(6.9)$ & $12(17.1)$ & & $3(6.1)$ & $8(16.3)$ & \\
\hline IIIA & $15(10.4)$ & $8(11.4)$ & & $5(10.2)$ & 7 (14.3) & \\
\hline IIIB & $15(10.4)$ & $10(14.3)$ & & $5(10.2)$ & 7 (14.3) & \\
\hline IVA & $8(5.6)$ & $4(5.7)$ & & $2(4.0)$ & $4(8.2)$ & \\
\hline
\end{tabular}

T-RAMIE, totally robot-assisted minimally invasive esophagectomy; H-RAMIE, hybrid robot-assisted minimally invasive esophagectomy; LN, lymph node; GIST, gastrointestinal stromal tumor.

clinical outcomes of esophageal cancer surgery. Therefore, we think there could be a difference with the results by Mariette et al. (15) which compared hybrid MIE with OE. The large randomized clinical trial comparing hybrid and total MIE should be conducted to examine the clinical benefit of adding minimally invasive abdominal procedures to thoracic procedures.

There are several other potential advantages of robotic abdominal surgery in addition to the clinical benefits that should be addressed. First, as there are an increased number of esophageal cancer patients who undergo neoadjuvant chemoradiation therapy, the surgical advantages afforded by robotic platforms might be beneficial for more meticulous tissue dissection. Second, the conduct of the operation can be greatly elevated because every single movement in robotic surgery, including the camera and assistance arm, is under direct control of the surgeon. Third, challenging surgical techniques and the long learning curves of conventional MIE can be overcome by many beneficial technologies provided by robotic surgical platforms. Last, the benefits to the surgeon in terms of ease and simplification of self-orchestrated operative performance and potential decrease in chronic work-related trauma and injuries, particularly involving long and complex operations, 
Table 4 Early clinical outcomes

\begin{tabular}{|c|c|c|c|c|c|c|}
\hline Variables & \multicolumn{3}{|c|}{ Unmatched groups } & \multicolumn{3}{|c|}{ Matched groups } \\
\hline 30-day mortality & 0 & $1(1.4)$ & 0.327 & 0 & $1(2.0)$ & 0.317 \\
\hline 90-day mortality & $1(0.7)$ & $3(4.3)$ & 0.104 & 0 & $3(6.1)$ & 0.083 \\
\hline Complications & $86(59.7)$ & $43(61.4)$ & 0.811 & $31(63.3)$ & $31(63.3)$ & 1.000 \\
\hline Respiratory complications & $15(10.4)$ & $5(7.1)$ & 0.440 & $5(10.2)$ & $5(10.2)$ & 1.000 \\
\hline Leakage & $9(6.3)$ & $7(10.0)$ & 0.328 & $3(6.1)$ & $5(10.2)$ & 0.480 \\
\hline Abdomen-related complications & $13(9.0)$ & 9 (12.9) & 0.297 & $4(8.2)$ & $7(14.3)$ & 0.366 \\
\hline Chyloperitoneum & $9(6.3)$ & $5(7.1)$ & & $4(8.2)$ & $3(6.1)$ & \\
\hline Bleeding & 0 & $1(1.4)$ & & 0 & $1(2.0)$ & \\
\hline Septic colitis & 0 & $1(1.4)$ & & 0 & $1(2.0)$ & \\
\hline Colon infarction & $1(0.7)$ & 0 & & 0 & 0 & \\
\hline Length of stay (median, range) & 13 days $(6-244)$ & 13.5 days $(7.0-63.0)$ & 0.908 & 14 days (8-244) & 14 days (7-63) & 0.936 \\
\hline
\end{tabular}

T-RAMIE, totally robot-assisted minimally invasive esophagectomy; H-RAMIE, hybrid robot-assisted minimally invasive esophagectomy.
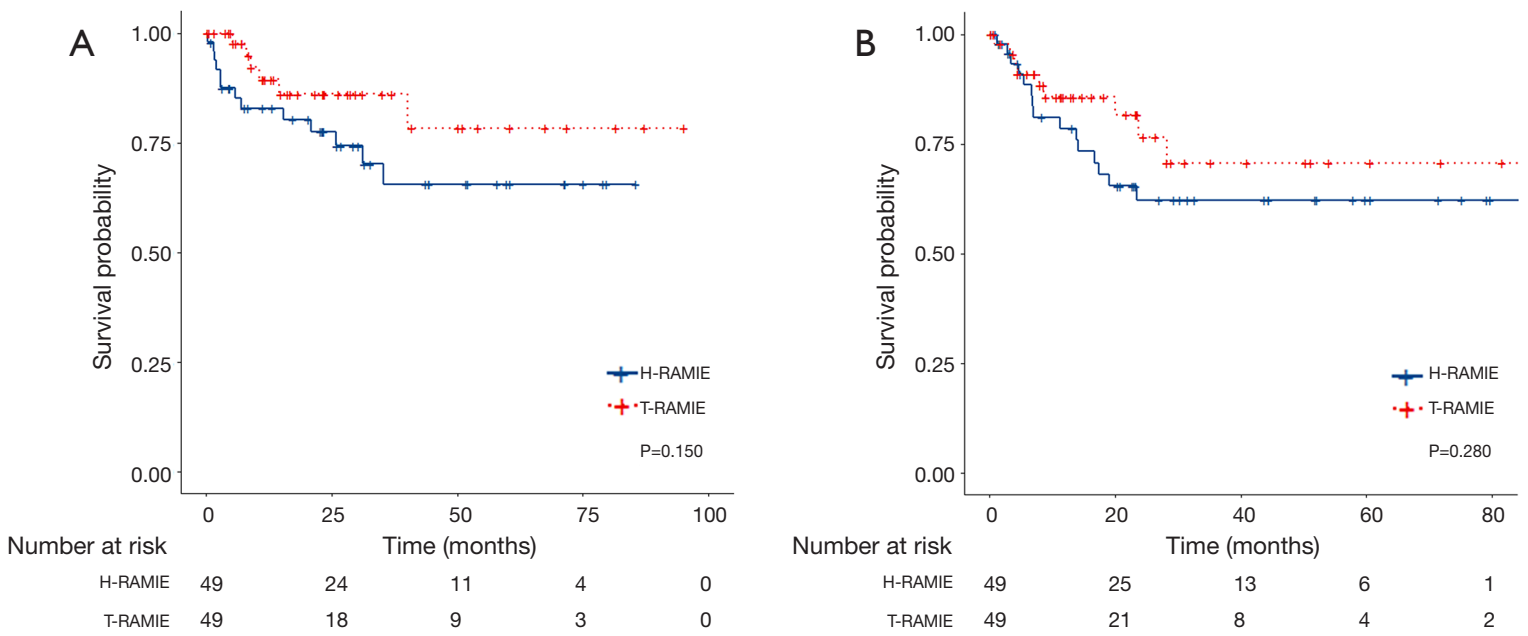

Figure 1 Long-term clinical outcome of the matched cohort. (A) Overall survival and (B) recurrence-free survival of the matched cohort. H-RAMIE, hybrid robotic minimally invasive esophagectomy; T-RAMIE, total robotic minimally invasive esophagectomy.

may be significant.

There are several limitations of this study that must be acknowledged. First, there might be a selection bias because this study is limited by its retrospective nature without randomization. There was a trend that the patients with more advanced esophageal cancer underwent H-RAMIE in the unmatched cohort. We conducted propensity score matching to minimize selection bias; however, the H-RAMIE group included more patients with advanced pathological stage. Additionally, uncontrolled bias might 
still exist due to its retrospective nature. Second, there might be a learning curve effect. The study included patients who underwent surgery within a relatively long study period. Experience of the surgeons might be an important factor for the clinical outcome of the patients (25). Therefore, uncontrolled heterogeneity, such as technical variability or changes in postoperative care protocol, might exist in both groups.

Conclusively, we reported favorable early and longterm clinical outcomes of T-RAMIE, and the overall outcome was comparable with that following H-RAMIE. Because T-RAMIE showed a low tendency for early operative mortality, as well as a very low conversion rate, and because robotic surgical systems provide a surgeonfriendly operative environment, T-RAMIE should be considered a safe alternative to open stomach mobilization and abdominal LN dissection.

\section{Acknowledgments}

None.

\section{Footnote}

Conflicts of Interest: The authors have no conflicts of interest to declare.

Ethical Statement: The authors are accountable for all aspects of the work in ensuring that questions related to the accuracy or integrity of any part of the work are appropriately investigated and resolved. The study protocol was reviewed by the Institutional Review Board and approved as a minimal risk retrospective study (Approval No. H-1905-137-1035) that did not require individual consent according to the institutional guidelines for consent waivers.

\section{References}

1. Birkmeyer JD, Siewers AE, Finlayson EV, et al. Hospital volume and surgical mortality in the United States. N Engl J Med 2002;346:1128-37.

2. Hulscher JB, van Sandick JW, de Boer AG, et al. Extended transthoracic resection compared with limited transhiatal resection for adenocarcinoma of the esophagus. N Engl J Med 2002;347:1662-9.

3. Biere SS, van Berge Henegouwen MI, Maas KW, et al. Minimally invasive versus open oesophagectomy for patients with oesophageal cancer: a multicentre, open-label, randomised controlled trial. Lancet 2012;379:1887-92.

4. Nafteux P, Moons J, Coosemans W, et al. Minimally invasive oesophagectomy: a valuable alternative to open oesophagectomy for the treatment of early oesophageal and gastro-oesophageal junction carcinoma. Eur J Cardiothorac Surg 2011;40:1455-65.

5. Luketich JD, Pennathur A, Awais O, et al. Outcomes after minimally invasive esophagectomy: review of over 1000 patients. Ann Surg 2012;256:95-103.

6. Luketich JD, Alvelo-Rivera M, Buenaventura PO, et al. Minimally invasive esophagectomy: outcomes in 222 patients. Ann Surg 2003;238:486-94.

7. Park SY, Kim DJ, Yu WS, et al. Robot-assisted thoracoscopic esophagectomy with extensive mediastinal lymphadenectomy: experience with 114 consecutive patients with intrathoracic esophageal cancer. Dis Esophagus 2016;29:326-32.

8. Park SY, Kim DJ, Do YW, et al. The oncologic outcome of esophageal squamous cell carcinoma patients after robot-assisted thoracoscopic esophagectomy with total mediastinal lymphadenectomy. Ann Thorac Surg 2017;103:1151-7.

9. Park S, Hwang Y, Lee HJ, et al. Comparison of robotassisted esophagectomy and thoracoscopic esophagectomy in esophageal squamous cell carcinoma. J Thorac Dis 2016;8:2853-61.

10. van der Sluis PC, van der Horst S, May AM, et al. Robot-assisted minimally invasive thoracolaparoscopic esophagectomy versus open transthoracic esophagectomy for resectable esophageal cancer: a randomized controlled trial. Ann Surg 2019;269:621-30.

11. Boone J, Schipper M, Moojen W, et al. Robot-assisted thoracoscopic oesophagectomy for cancer. Br J Surg 2009;96:878-86.

12. van der Sluis PC, Ruurda JP, Verhage RJ, et al. Oncologic Long-Term Results of Robot-Assisted Minimally Invasive Thoraco-Laparoscopic Esophagectomy with Two-Field Lymphadenectomy for Esophageal Cancer. Ann Surg Oncol 2015;22:1350-6.

13. Motoyama S, Sato Y, Wakita A, et al. Extensive Lymph Node Dissection Around the Left Laryngeal Nerve Achieved With Robot-assisted Thoracoscopic Esophagectomy. Anticancer Res 2019;39:1337-42.

14. van Hillegersberg R, Boone J, Draaisma WA, et al. First experience with robot-assisted thoracoscopic esophagolymphadenectomy for esophageal cancer. Surg 
Endosc 2006;20:1435-9.

15. Mariette C, Markar SR, Dabakuyo-Yonli TS, et al. Hybrid minimally invasive esophagectomy for esophageal cancer. N Engl J Med 2019;380:152-62.

16. Amin MB, Edge SB. AJCC cancer staging manual: Springer; 2017.

17. Mellow MH, Pinkas H. Endoscopic laser therapy for malignancies affecting the esophagus and gastroesophageal junction: analysis of technical and functional efficacy. Arch Intern Med 1985;145:1443-6.

18. Oken MM, Creech RH, Tormey DC, et al. Toxicity and response criteria of the Eastern Cooperative Oncology Group. Am J Clin Oncol 1982;5:649-55.

19. Dindo D, Demartines N, Clavien PA. Classification of surgical complications: a new proposal with evaluation in a cohort of 6336 patients and results of a survey. Ann Surg 2004;240:205-13.

20. Low DE, Alderson D, Cecconello I, et al. International Consensus on Standardization of Data Collection for Complications Associated With Esophagectomy: Esophagectomy Complications Consensus Group (ECCG). Ann Surg 2015;262:286-94.

Cite this article as: $\mathrm{Na} \mathrm{KJ}$, Park S, Park IK, Kim YT, Kang CH. Outcomes after total robotic esophagectomy for esophageal cancer: a propensity-matched comparison with hybrid robotic esophagectomy. J Thorac Dis 2019;11(12):53105320. doi: $10.21037 /$ jtd.2019.11.58
21. Chao YK, Hsieh MJ, Liu YH, et al. Lymph node evaluation in robot-assisted versus video-assisted thoracoscopic esophagectomy for esophageal squamous cell carcinoma: a propensity-matched analysis. World J Surg 2018;42:590-8.

22. Lee JH, Park DJ, Kim HH, et al. Comparison of complications after laparoscopy-assisted distal gastrectomy and open distal gastrectomy for gastric cancer using the Clavien-Dindo classification. Surg Endosc 2012;26:1287-95.

23. Kim W, Kim HH, Han SU, et al. Decreased morbidity of laparoscopic distal gastrectomy compared with open distal gastrectomy for stage I gastric cancer. Ann Surg 2016;263:28-35.

24. Woo Y, Hyung WJ, Pak KH, et al. Robotic gastrectomy as an oncologically sound alternative to laparoscopic resections for the treatment of early-stage gastric cancers. Arch Surg 2011;146:1086-92.

25. Park S, Hyun K, Lee HJ, et al. A study of the learning curve for robotic oesophagectomy for oesophageal cancer. Eur J Cardiothorac Surg 2017;53:862-70. 